\title{
Pion Production under the Action of Intense Ultrashort Laser Pulse on a Solid Target
}

\author{
V. Yu. Bychenkovi, *, Y. Sentoku², S. V. Bulanov ${ }^{3}$, K. Mima², \\ G. Mourou ${ }^{4}$, and S. V. Tolokonnikov ${ }^{5}$ \\ ${ }^{1}$ Lebedev Physical Institute, Russian Academy of Sciences, Leninskiu pr. 53, Moscow, 119991 Russia \\ *e-mail: bychenk@sci.lebedev.ru \\ ${ }^{2}$ Institute of Laser Engineering, University of Osaka, 565-0871 Osaka, Japan \\ ${ }^{3}$ Institute of General Physics, Russian Academy of Sciences, ul. Vavilova 38, Moscow, 119991 Russia \\ ${ }^{4}$ Center for Ultrafast Optical Science, University of Michigan, 48109-2099 Ann Arbor, Michigan, USA \\ ${ }^{5}$ Kurchatov Institute Russian Research Centre, pl. Kurchatova 1, Moscow, 123182 Russia
}

Received October 22, 2001; in final form November 14, 2001

Two-dimensional "particle-in-cell" modeling was carried out to determine the laser intensity threshold for pion production by protons accelerated by the relativistically strong short laser pulses acting on a solid target. The pion production yield was determined as a function of laser intensity. ( 2001 MAIK "Nauka/Interperiodica".

PACS numbers: 52.38.Kd; 25.40.Qa

Generation of energetic ions by ultrashort laser pulses interacting with plasma has been the object of active studies over recent years [1-9]. This is primarily dictated by the necessity of understanding the mechanisms of ion acceleration as a result of the interaction of laser radiation with plasma, including the quantitative description of the characteristics of these mechanisms. The problem of ion acceleration is among the key problems in various applications of high-power lasers such as fast ignition for laser fusion, injection of fast particles, and fabrication of radioactive sources in medicine and nuclear physics. Due to the effective acceleration of ions in a laser field, they can initiate nuclear reactions.

At the present level of energies, ultrashort pulsed lasers are capable of initiating various nuclear reactions, such as nuclear fusion, fission, pickup, stripping, charge-transfer, and radiative capture [1]. Nuclear reactions in the collisions of laser-accelerated ions with a nuclear target were observed experimentally at energies from several $\mathrm{MeV}$ to tens of $\mathrm{MeV}$ [5-7]. The detection of protons with an energy of $60 \mathrm{MeV}$ at a laser intensity of $3 \times 10^{20} \mathrm{~W} / \mathrm{cm}^{2}[5]$ and the progress in laser energy buildup allow one to assume that the pion-production threshold of $140 \mathrm{MeV}$ will be surmounted in the near future. The generation of such energies in the laboratory, rather than on accelerators, may have a profound effect on the priorities in laser technology and nuclear research techniques. With these prospects in mind, it is profitable to estimate quantitatively the pion yield as a function of laser parameters and determine the corresponding laser intensity threshold. In this communication, the results of relativistic two-dimensional particlein-cell (PIC) modeling are presented.
Note that the pion production by a short laser pulse and the pion yield have already been discussed in [10]. This process was associated with the formation of gamma-ray quanta, which initiated pion photoproduction. The formation of gamma-ray quanta is caused by the bremsstrahlung of laser-accelerated electrons, after which gamma-ray quanta interact with nuclei to produce $\Delta$ isobars that decay with pion emission. This transformation chain is highly inefficient because of its electromagnetic nature, as contrasted to the strong interactions of protons and nuclei discussed below.

In our computations, we modeled the interaction of a short 300-fs linearly polarized pulse with a solid $5-\mu \mathrm{m}$-thick foil representing a uniform plasma layer with a density forty times higher than its critical value. Laser radiation with a wavelength of $1 \mu \mathrm{m}$ was incident normally to the foil surface and propagated along the $X$ direction of the modeling plane $X Y$ of size $50 \times 10 \mu \mathrm{m}$. The focusing spot size was $3 \mu \mathrm{m}$, and the computations were carried out for several values of laser intensity $I$ in the range from $2 \times 10^{20}$ to $5 \times 10^{21} \mathrm{~W} / \mathrm{cm}^{2}$, which corresponded to a relativistically strong laser field $a \gg 1$, where $a=e A / m c^{2}$ is the amplitude of the normalized vector potential $A$, and $c, e$, and $m$ are the light velocity, the electron charge, and the electron mass, respectively. For definiteness, a two-component plasma composed of $50 \% \mathrm{H}$ and $50 \% \mathrm{D}$ was considered. To make the model as realistic as possible, a 30- $\mu \mathrm{m}$-thick layer of rarefied plasma was placed ahead of the foil, and its density was taken to be constant and equal to $10^{21} \mathrm{~cm}^{-3}$. The point is that the laser pulse is nonideal and preceded by a prepulse at experimental power levels much higher than $1 \mathrm{TW}$, leading to the appearance of a rather extended 
40- $\mu$ m-thick "preplasma" layer at the target surface before the pulse peak reaches it [5]. Such a underdense plasma strongly affects the character of interaction between the pulse and the target. The plasma proved to be nontransparent for intensities $I \lesssim 5 \times 10^{21} \mathrm{~W} / \mathrm{cm}^{2}$. We also carried out the computations for an intensity of $1.2 \times 10^{22} \mathrm{~W} / \mathrm{cm}^{2}$, for which the relativistic transparency effect arose and a substantial fraction of laser radiation passed through the target.

The preplasma electrons with relativistic energies are accelerated in the forward direction, pass through the thin foil, and create a strong ion-accelerating electrostatic field. The formation of the ion-accelerating electric field is a rather complicated process, because the generation of quasi-static magnetic fields and the formation of a region with an excess of positive electric charge may play an important role in this case [11]. Without going into details (see [11]), note that it is the generation of hot electrons which is at the origin of the ion acceleration. For the relativistic intensities, the main part of the laser energy transforms into the energy of hot electrons $[5,12]$. Then the kinetic energy $T_{h}$ of hot electrons transforms into the energy of electrostatic field accelerating ions. Accordingly, one should expect that the energy $\epsilon_{i}$ of the latter is on the order of the energy of hot electrons; i.e., $\epsilon_{i} \sim Z T_{h}$. Our computations suggest that, indeed, the characteristic proton energy varies in proportion to the temperature of hot electrons with changing laser intensity. The deuteron acceleration is suppressed appreciably, as compared to the proton acceleration. Due to higher mobility, protons are accelerated first, as a result of which the electric field behind them decreases and less efficiently accelerates deuterons following them. As a result, the calculated deuteron energy did not exceed one-fourth of the proton energy. This effect was observed experimentally in [7], where the proton and deuteron energies were equal to 9 and $2 \mathrm{MeV}$, respectively.

When passing through the preplasma, laser radiation was canalized, due to the relativistic self-focusing effect, to efficiently accelerate electrons. Qualitatively, their energy distribution corresponded to the Maxwell distribution with temperature $T_{h}$ satisfying approximately a root law $T_{h} \propto \sqrt{I}$ with respect to the laser intensity. At the same time, the electron motion proves to be highly complicated (stochastic) because of the complex structure of the fields, including the reflected laser wave and the fields generated in plasma. The behavior $\propto \sqrt{I}$ of the hot electron temperature corresponds qualitatively to the scaling law for the electron energy accumulation in a relativistically strong electromagnetic wave propagating in plasma with the phase velocity $\omega / k$ higher than the light velocity $c k \leqslant \omega$. The dependence of the dimensionless electron kinetic energy $\gamma$ on the laser intensity in the ultrarelativistic limit $a \gg 1$ can be estimated using the well-known inte- grals of motion [13] for an electron in an electromagnetic field,

$$
\frac{p_{x}}{m c}=\frac{c k}{\omega} \gamma, \quad \frac{p_{y}}{m c}=a, \quad \gamma=\sqrt{\frac{p_{x}^{2}}{m^{2} c^{2}}+\frac{p_{y}^{2}}{m^{2} c^{2}}},
$$

which are written for an initially quiescent electron. According to Eq. (1), $\gamma=a \omega / \sqrt{\omega^{2}-c^{2} k^{2}} \propto \sqrt{I}$. This scaling law is similar to the one obtained from the ponderomotive potential [14] $T_{h}=m c^{2}\left(\sqrt{1+a^{2}}-1\right) \simeq$ $m c^{2} a$. However, contrary to the latter, it includes a contribution from the longitudinal electron momentum, which is not smaller than the transverse momentum and, hence, adds to the energy. This is confirmed by our computations showing that the formula for $T_{h}$ from [14] underestimates the temperature of hot electrons.

We studied the ion energy as a function of laser intensity in the absence of preplasma and established that this dependence was also close to the root law, as in the case with preplasma, but the energy was slightly lower. Therefore, the presence of preplasma is quite important, because it enhances the generation efficiency of both fast electrons and ions. In Fig. 1, the energy spectra of the forward accelerated protons are shown for three values of laser intensity $I \leq 5 \times$ $10^{21} \mathrm{~W} / \mathrm{cm}^{2}$. As the energy increases, the high-energy tail shown in this figure transforms into a plateaulike distribution with an abrupt cutoff. Such a plateaulike distribution with energy cutoff was observed experimentally in $[4-7,15]$, and it is typical of the electrostatic ion-acceleration mechanism. The cutoff corresponds to the maximal potential difference created by hot electrons. At a higher intensity of $1.2 \times 10^{22} \mathrm{~W} / \mathrm{cm}^{2}$, plasma becomes relativistically transparent, and the inductive electric field arising on the rear side of the target due to the generated magnetic field plays a substantial part in the ion acceleration [16].

Evidently, once the cutoff in the proton spectrum exceeds $140 \mathrm{MeV}$, protons can produce $\pi^{+}$in a sub-

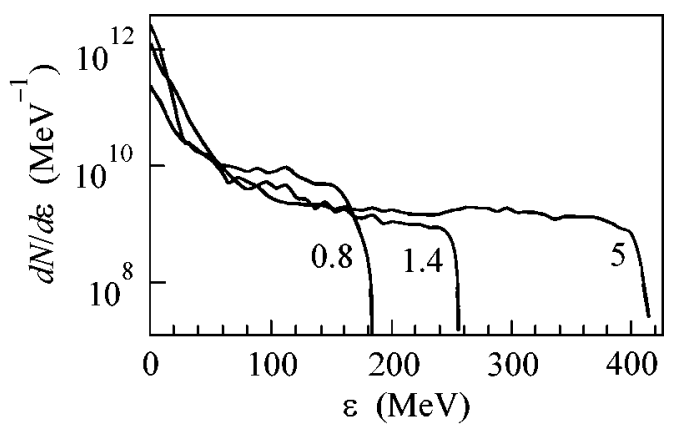

Fig. 1. Energy distribution of the number of forward accelerated protons at different laser intensities $I$. The values of $I$ in $10^{21} \mathrm{~W} / \mathrm{cm}^{2}$ are indicated near the corresponding curves. 


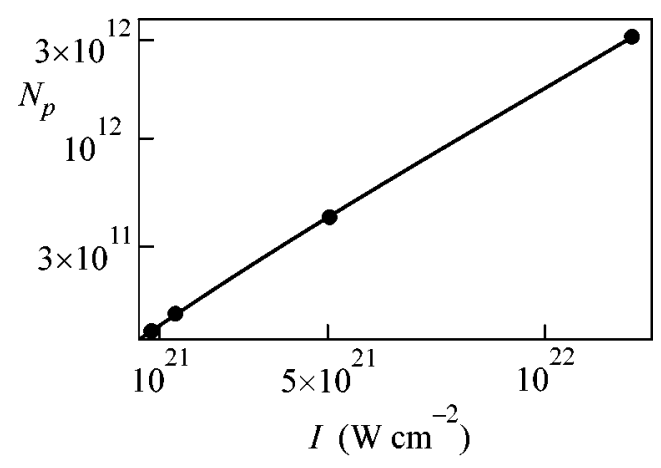

Fig. 2. The number of forward accelerated protons with energy exceeding $140 \mathrm{MeV}$ vs. laser intensity.

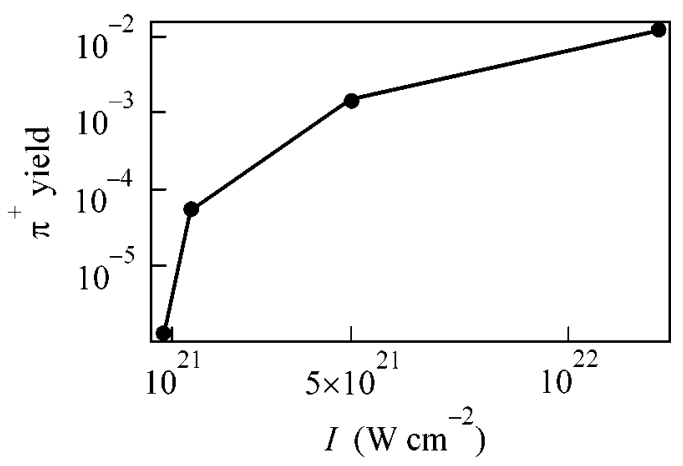

Fig. 3. Pion yield (per a proton) as a function of pulse intensity. The curve corresponds to the $\pi^{+}$production in the proton collisions with the $\mathrm{C}$ nuclei.

stance behind the foil. The number $N_{p}$ of accelerated protons with energy higher than $140 \mathrm{MeV}$ is shown in Fig. 2 as a function of laser intensity. The accelerated protons will react with the nuclei of the substance in a layer of thickness on the order of proton stopping range, which is mainly determined by the ionization loss. The number of produced pions is lesser than the number of accelerated protons in a ratio equal to the ratio of proton stopping range to the proton path associated with $\pi^{+}$-production reaction. Quantitatively, this number,

$$
N_{\pi}=\frac{1}{M_{a}} \int_{0}^{\infty} d \epsilon \frac{d N}{d \epsilon} \int_{0}^{\epsilon} d \epsilon^{\prime} \frac{\sigma\left(\epsilon^{\prime}\right)}{W\left(\epsilon^{\prime}\right)},
$$

is determined by the cross section $\sigma$ for reaction $p+$ $A \longrightarrow \pi^{+} \ldots$, where $A$ is a nucleus with mass $M_{a}$, the stopping loss $W(\mathrm{MeV} \mathrm{cm} / \mathrm{g})$ of protons in the substance $a$, and the pion number distribution in energy $d N / d \epsilon$.

We considered carbon and germanium targets as examples of light and heavy nuclear targets, respectively. Pion yield (2) per one proton is presented for the carbon nuclei in Fig. 3, where the data for $\sigma$ and $W$ are taken from [17]. Despite the fact that the reaction cross section for pion production far above the threshold behaves as $A^{2 / 3}$ ( $A$ is the nucleus atomic number), the overlap integral in Eq. (2) shows little dependence on the atomic number. Our calculations showed that the pion yield in germanium differs from its value in carbon by only $9-15 \%$.

Our calculations can be used to predict the laserintensity threshold for pion production. This threshold is clearly seen in Fig. 3 at $I_{\text {th }} \sim 10^{21} \mathrm{~W} / \mathrm{cm}^{2}$. At $I=5 \times$ $10^{21} \mathrm{~W} / \mathrm{cm}^{2}$, the pion production yield is $10^{-3}$. For $4 \times 10^{11}$ accelerated protons, this provides a value of $4 \times 10^{8}$ particles per shot for the total $\pi^{+}$yield, which is five orders of magnitude higher than the photopion yield obtained under similar conditions in [10]. Pions can be most simply identified by the standard method from the $\pi \mu$ decay, because the lifetime of $\pi^{+}$is short, $\tau_{\pi} \simeq 26 \mathrm{~ns}$. At a pulse repetition rate of $1 \mathrm{kHz}$, the laser muon production will offer advantages over the conventional accelerator methods and provide a muon flux of $10^{14}-10^{15} \mathrm{~s}^{-1}$.

At first glance, the value of $\sim 10^{21} \mathrm{~W} / \mathrm{cm}^{2}$ found above for the pion production threshold can be attained using low-energy lasers, provided that the pulse duration equals several femtoseconds. This is quite possible in current laser techniques. However, there is a fundamental limitation that requires a considerably longer pulse duration. The reason is that, due to a large mass $M$ of ions (protons), they are accelerated in a certain finite time $\tau_{i}$, which is determined by the condition $\tau_{i} \sim$ $d / v$, where $v$ is the ion velocity, $v \sim \sqrt{T_{h} / M}$, and $d$ is the length of accelerating interval $d \sim \lambda_{D}$, where $\lambda_{D} \sim$ $\left(T_{h} / e^{2} n_{h}\right)^{1 / 2}$ is the Debye radius of hot electrons with density $n_{h}$; i.e., $\tau_{i} \sim \sqrt{M / e^{2} n_{h}}$. Taking a value on the order of critical density in estimating the density $n_{h}$ of hot electrons, one obtains $\tau_{i} \sim 100 \mathrm{fs}$. Since the chargeseparation field is sustained by the laser, its duration must exceed $\tau_{i}$, in order that the protons have time to accelerate to the maximal energy determined by the charge-separation field. On the other hand, the smallest attainable focal spot size is equal to several microns for high-power lasers. Hence, it follows that, to produce pions, the laser output should be as high as several tens of joules.

In the above examples of pion production in the proton collisions with nuclei, the pion distribution is virtually isotropic. At the same time, for the hydrogen-containing nuclear targets, where pions may be produced in the $p-p$ collisions, one can expect the generation of directed pion and neutrino fluxes, provided that the proton energy far exceeds $1 \mathrm{GeV}$, i.e., at $I>10^{23} \mathrm{~W} / \mathrm{cm}^{2}$ according to the scaling law discussed above. The lifetime of pions with energies up to several $\mathrm{GeV}$ is considerably larger than $\tau_{\pi}$. This opens the way to their subse- 
quent acceleration by the laser plasma method of particle acceleration in a underdense plasma [18] to produce ultrahigh-energy pions, which are, as yet, present only in cosmic rays. However, contrary to the latter, the laser generation of ultrahigh-energy pions is predictable.

In summary, numerical modeling of the interaction of a short relativistically strong laser pulse with plasma produced by the irradiation of a foil has been carried out in this work to investigate the generation of protons with energies of hundreds of $\mathrm{MeV}$ and pion production in proton collisions with light and heavy nuclei. The laser intensity threshold is determined at which pion production should be expected, and the pion yield is obtained as a function of laser intensity. This effect corresponds to the nuclear regime of interaction between the radiation of a high-power laser and a substance. The mastering of this effect will allow the development of a new exploratory base for high-energy physics.

This work was supported by the Russian Foundation for Basic Research, project no. 00-02-16063.

\section{REFERENCES}

1. V. Yu. Bychenkov, V. T. Tikhonchuk, and S. V. Tolokonnikov, Zh. Éksp. Teor. Fiz. 115, 2080 (1999) [JETP 88, 1137 (1999)].

2. S. V. Bulanov, T. Zh. Esirkerov, F. Califano, et al., Pis'ma Zh. Éksp. Teor. Fiz. 71, 593 (2000) [JETP Lett. 71, 407 (2000)].

3. A. Maksimchuk, S. Cu, K. Flippo, et al., Phys. Rev. Lett. 84, 4108 (2000).

4. E. L. Clark, K. Krushelnick, M. Zepf, et al., Phys. Rev. Lett. 85, 1654 (2000).
5. S. P. Hatchett, C. G. Brown, Th. E. Cowan, et al., Phys. Plasmas 7, 2076 (2000); R. A. Snavely, M. H. Key, S. P. Hatchett, et al., Phys. Rev. Lett. 85, 2945 (2000).

6. K. Krushelnick, E. L. Clark, M. Zepf, et al., Phys. Plasmas 7, 2055 (2000); E. L. Clark, K. Krushelnick, J. R. Davies, et al., Phys. Rev. Lett. 84, 670 (2000).

7. K. Nemoto, A. Maksimchuk, S. Banerjee, et al., Appl. Phys. Lett. 78, 595 (2001).

8. S. C. Wilks, A. B. Langdon, T. E. Cowan, et al., Phys. Plasmas 8, 542 (2001).

9. A. Pukhov, Phys. Rev. Lett. 86, 3562 (2001).

10. S. Karsch, D. Habs, T. Schatz, et al., Laser Part. Beams 17, 565 (1999).

11. A. V. Kuznetsov, T. Zh. Esirkepov, F. F. Kamenets, and S. V. Bulanov, Fiz. Plazmy 27, 225 (2001) [Plasma Phys. Rep. 27, 211 (2001)].

12. K. B. Wharton, S. P. Hatchett, S. C. Wilks, et al., Phys. Rev. Lett. 81, 822 (1998).

13. V. A. Buts and A. V. Buts, Zh. Éksp. Teor. Fiz. 110, 818 (1996) [JETP 83, 449 (1996)].

14. S. C. Wilks, W. L. Kruer, M. Tabak, and A. B. Langdon, Phys. Rev. Lett. 69, 1383 (1992).

15. G. S. Sarkisov, V. Yu. Bychenkov, V. N. Novikov, et al., Phys. Rev. E 59, 7042 (1999).

16. Y. Sentoku, T. V. Liseikina, T. Zh. Esirkepov, et al., Phys. Rev. E 62, 7271 (2000).

17. B. Jakobsson, Yu. Murin, and V. G. Khlopin, Nucl. Phys. A 630, 184 (1998); http://www.triumf.ca/triumf-handbooks; http://physics.nist.gov/Star.

18. T. Tajima and J. M. Dawson, Phys. Rev. Lett. 43, 267 (1979).

Translated by V. Sakun

JETP LETTERS Vol. $74 \quad$ No. $12 \quad 2001$ 